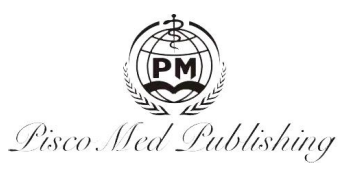

\title{
Original Research Article \\ Research on Supply Chain Resilience of Agricultural Products Based on AHP-FCE Model
}

\author{
Wenyu Dong \\ Logistics Research Center, Shanghai Maritime University, Shanghai 201306, China
}

\begin{abstract}
As the proportion of risks in the supply chain of agricultural products increases gradually, it is of far-reaching significance to improve the supply chain resilience to resist the risk interference. Based on the research on the factors affecting the resilience of agricultural products supply chain, the AHP-FCE method was used to evaluate the resilience of agricultural products supply chain. Through qualitative analysis and quantitative calculation, the fuzzy comprehensive evaluation of agricultural product supply chain resilience is carried out. The results show that the reliability of suppliers, the number of transport vehicles and the risk control ability of employees are the main factors restricting the resilience of agricultural supply chain. Finally, it provides theoretical basis for relevant enterprises and institutions to improve the resilience of their agricultural supply chain through case analysis and verification.
\end{abstract}

Keywords: Agricultural Products; Supply Chain Elasticity; Analytic Hierarchy Process - Fuzzy Comprehensive Evaluation Method

\section{Introduction}

China is a large agricultural country. According to data from the National Bureau of Statistics, the total agricultural output value in 2019 increased by 7.5\% year-on-year. The affected area was 19,257 thousand hectares, and the disaster-affected area was 791.3 thousand hectares. It is difficult to control the random fluctuation of agricultural product supply. With the increasing dependence of society on the demand for agricultural products, the risks faced by the agricultural product supply chain have gradually increased. To deal with the risks of the agricultural product supply chain and improve the circulation efficiency of agricultural products, it is necessary to enhance the resilience of the agricultural product supply chain.

The frequent occurrence of emergencies continues to cause the supply chain to be interrupted, which has aroused the attention of many scholars to the elasticity of the supply chain (Pettit et al. 2013; Soni et al., 2014). Hosseini et al. (2012) used data envelopment analysis to comprehensively and partially evaluate supply chain resilience. Joshua Aboah et al. (2019) takes adaptability, flexibility, collaboration and other aspects as the starting point, adopts the citation network analysis method to study the factors that affect the elasticity of the agricultural supply chain, and builds the elasticity evaluation model. Hosseini et al. (2019) studied ways to improve supply chain flexibility, such as good supply chain flexibility, complete transportation infrastructure, and risk mitigation inventory.

At present, there are few research results on constructing a comprehensive evaluation model of supply chain resilience with agricultural product supply chain as the research object. Therefore, this article considers many factors and systematically establishes an evaluation index system for agricultural supply chain resilience, based on the comprehensive application of AHPFCE method. Point out the method of constructing the elasticity evaluation model of agricultural product supply chain, verify the effectiveness of this method through case study, and provide theoretical basis for relevant enterprises to improve the elasticity of agricultural product supply chain.

\section{AHP-FCE model}

\subsection{Build a ladder hierarchy model}

From the perspective of the elasticity of the agricultural product supply chain, this paper follows the principles of science, standardization, and refinement to construct an evaluation index system for the elasticity of the agricultural product supply chain, as shown in Table 1.

\begin{tabular}{r|r|r}
$\operatorname{Target}$ layer $(A)$ & $\operatorname{Criterion~layer}\left(A_{i}\right)$ & $\operatorname{Index} \operatorname{laver}\left(A_{i j}\right)$ \\
\hline
\end{tabular}




\begin{tabular}{|c|c|c|}
\hline \multirow{16}{*}{$\begin{array}{c}\text { Evaluation index } \\
\text { system of agricultural } \\
\text { product supply Chain } \\
\text { elasticity }(A)\end{array}$} & \multirow{4}{*}{$\begin{array}{l}\text { Infrastructure } \\
\left(A_{1}\right)\end{array}$} & Number of transport vehicles $\left(A_{11}\right)$ \\
\hline & & Intelligent proportion of equipment ( $\left.A_{12}\right)$ \\
\hline & & Cold chain temperature monitoring facility $\left(A_{13}\right)$ \\
\hline & & Storage facility $\left(A_{14}\right)$ \\
\hline & \multirow{5}{*}{ Supply and demand ( $\left.A_{2}\right)$} & Number of suppliers $\left(A_{21}\right)$ \\
\hline & & Supplier reliability $\left(A_{22}\right)$ \\
\hline & & Core business outsourcing ratio ( $\left.A_{23}\right)$ \\
\hline & & Work efficiency $\left(A_{24}\right)$ \\
\hline & & Safety stock $\left(A_{25}\right)$ \\
\hline & \multirow{4}{*}{ Talent system $\left(A_{3}\right)$} & Risk identification ability $\left(A_{31}\right)$ \\
\hline & & Risk control ability $\left(A_{32}\right)$ \\
\hline & & Management level $\left(A_{33}\right)$ \\
\hline & & techinque level $\left(A_{34}\right)$ \\
\hline & \multirow{3}{*}{ Information system $\left(A_{4}\right)$} & Information network construction ( $\left.A_{41}\right)$ \\
\hline & & Accuracy of information $\left(A_{42}\right)$ \\
\hline & & Timeliness of information ( $\left.A_{43}\right)$ \\
\hline
\end{tabular}

Table 1. Evaluation index system of agricultural product supply chain elasticity

\subsection{Use AHP method to calculate weight vector}

\subsubsection{Construct a comparison discriminant matrix}

Based on the ladder hierarchy model in Table 1, using the Delphi expert consultation method, the comparison discriminant matrix $A_{i j}$ of 1-9 scale and its reciprocal is obtained through pairwise comparison. Aij scale of 1 means that factor $\mathrm{i}$ and factor $\mathrm{j}$

have the same importance. As the scale number increases, the importance of factor $i$ is gradually increasing compared to factor $j$.

\subsubsection{Weight vector calculation and consistency check}

Step1: Multiply the rows of the comparison discriminant matrix,

let $M_{i}=\prod_{j=1}^{n} A_{i j}, m_{i}=\sqrt[n]{M_{i}}, \quad W_{i}=\frac{m_{i}}{\sum_{i=1}^{n} m_{i}}$ Where, $i=1,2, \ldots n$

Step2: The maximum eigenvalue of matrix $A_{i j} \quad \lambda \max =\frac{1}{n} \sum_{i=1}^{n} \frac{W_{i}}{W_{i}}$

Step3: Seeking consistency test index $C I=\frac{\lambda \max -n}{n-1}$

Step4: Find the random consensus rate $C R=\frac{C I}{R I}$

Among them, $R I$ is the average random consistency index, and its value is shown in Table 3. If $C R<0.1$, pass the consistency test, otherwise, the value of the comparison discriminant matrix needs to be adjusted

Table 3. RI average random consistency index value

\begin{tabular}{ccccccc}
\hline $\mathrm{n}$ (Matrix order) & 1 & 2 & 3 & 4 & 5 & 6 \\
\hline $\mathrm{RI}$ & 0.00 & 0.00 & 0.52 & 0.89 & 1.12 & 1.26 \\
\hline
\end{tabular}

Step5: Calculate the total weight $W_{j}$ of each vector in the index layer, and its value is the index weight of this layer multiplied by the index weight of the previous layer, and the consistency test of the total weight.

\subsection{Establish fuzzy relation matrix}

(1) The factor set is the factors in the criterion layer and indicator layer in Table 1.

(2) Define the evaluation set $\mathrm{V}=\{$ excellent, good, medium, bad $\}$ is the evaluation of the factor set based on fuzzy theory by the evaluator.

(3) The weight set is the total weight $W_{j}$ of each vector in the index layer calculated and tested. 
(4) According to the evaluation statistics of the factor set, it is sorted out its grade, namely the degree of membership, and the fuzzy relationship matrix $R_{m \times n}$ is obtained. The value of the degree of membership $r_{m \times n}$ of each indicator is the number of the grades to which it belongs divided by the total number of surveys.

$$
\mathrm{R}_{\mathrm{m} \times \mathrm{n}}=\left(\begin{array}{cccc}
r_{11} & r_{12} & \cdots & r_{1 n} \\
r_{21} & r_{22} & \cdots & r_{2 n} \\
\vdots & \vdots & \vdots & \vdots \\
r_{m 1} & r_{m 2} & \ldots & r_{m n}
\end{array}\right)
$$

\subsection{Fuzzy comprehensive evaluation}

(1) Fuzzy comprehensive evaluation of criterion level $\mathrm{T}_{\mathrm{Ai}}=W i j \cdot \mathrm{R}_{\mathrm{m} \times \mathrm{n}}$

(2) Fuzzy comprehensive evaluation of target layer $T_{A}=W_{i} \cdot T_{A i}=\left(t_{1}, t_{2}, \ldots t_{n}\right)$

(3) According to the principle of maximum membership degree, the evaluation set to which the maximum value of $\mathrm{T}_{\mathrm{A}}$ belongs is the final evaluation result of the agricultural product supply chain.

\section{Case studies}

Taking Jiangxi Agricultural Products Supply Chain Co., Ltd. as an example, 10 experts from universities and enterprises are invited to construct the comparison judgment matrix of product supply chain elasticity through the pairwise comparison method. Use MATLAB to calculate, the results are shown in Table 4, Figure 1, and Table 5.

Table 4. Index calculation results of each matrix

\begin{tabular}{|c|c|c|c|c|c|}
\hline Matrix & Wi & $\lambda \max$ & CI & CR & $\begin{array}{c}\text { Whether to pass the } \\
\text { consistency test }\end{array}$ \\
\hline A-Ai & $(0.2485,0.4179,0.2245,0.1090)$ & 4.0457 & 0.0152 & 0.0171 & pass \\
\hline A1-Aij & $(0.4688,0.0953,0.1603,0.2766)$ & 4.0310 & 0.0103 & 0.0166 & pass \\
\hline A2-Aij & $(0.0955,0.4493,0.1910,0.1761,0.0881)$ & 5.2623 & 0.0659 & 0.0589 & pass \\
\hline A3-Aij & $(0.3038,0.4617,0.1013,0.1333)$ & 4.1320 & 0.0440 & 0.0494 & pass \\
\hline A4-Aij & $(0.5472,0.2631,0.1897)$ & 3.0183 & 0.0091 & 0.0022 & pass \\
\hline
\end{tabular}

Figure 1. The total weight of each indicator of the index layer.

Table 5. Index level index consistency test results

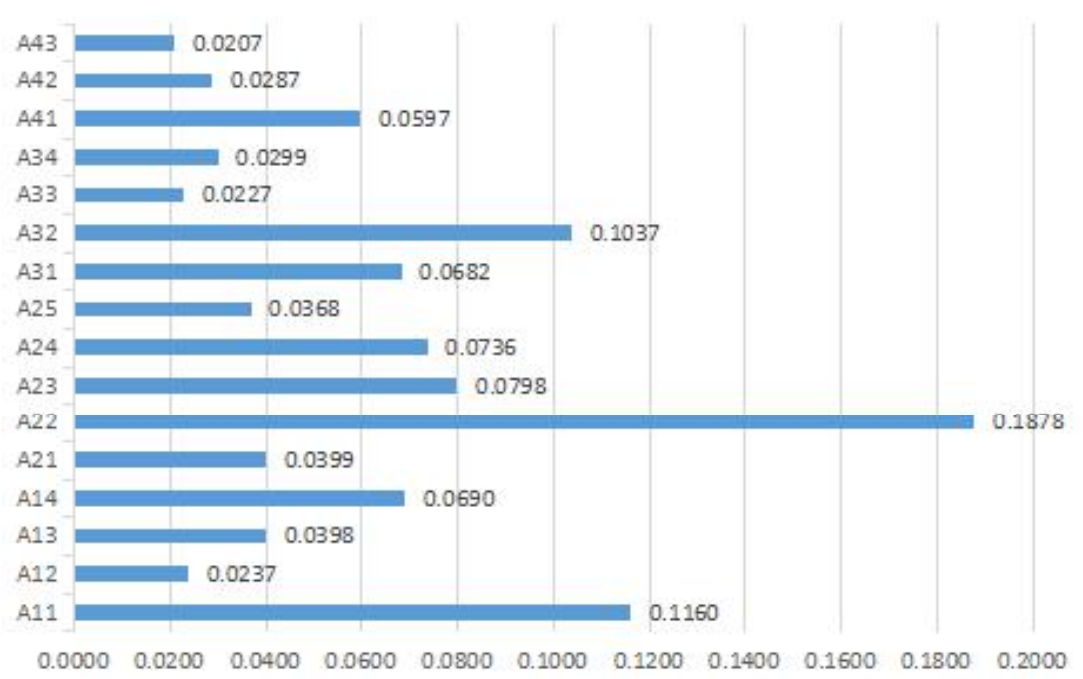

\begin{tabular}{cccc}
\hline $\mathrm{CI}=\sum$ AijCI & $\mathrm{RI}=\sum$ AijRI & $\mathrm{CR}=\mathrm{CI} / \mathrm{RI}$ & Whether to pass the consistency test \\
\hline 0.0261 & 0.9458 & 0.0275 & pass \\
\hline
\end{tabular}

In this paper, the company's employees and related experts were investigated, and 274 valid questionnaires of the evaluation levels of each index were obtained. The evaluation level table of each index and its corresponding fuzzy relationship matrix are shown in Table 6.

Table 6. Evaluation grade table of each index and its corresponding fuzzy relationship matrix

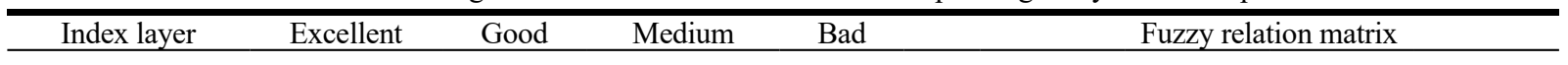




\begin{tabular}{|c|c|c|c|c|c|c|c|c|c|}
\hline A11 & 65 & 196 & 13 & 0 & & 0.2372 & 0.7153 & 0.0474 & 0.0000 \\
\hline A12 & 34 & 138 & 76 & 26 & \multirow{4}{*}{$\mathrm{R} 1$} & 0.1241 & 0.5036 & 0.2774 & 0.0949 \\
\hline A13 & 201 & 61 & 11 & 1 & & 0.7336 & 0.2226 & 0.0401 & 0.0036 \\
\hline A14 & 80 & 166 & 23 & 5 & & 0.2920 & 0.6058 & 0.0839 & 0.0182 \\
\hline A 21 & 175 & 88 & 9 & 2 & & 0.6387 & 0.3212 & 0.0328 & 0.0073 \\
\hline A 22 & 143 & 127 & 4 & 0 & \multirow{5}{*}{$\mathrm{R} 2$} & 0.5219 & 0.4635 & 0.0146 & 0.0000 \\
\hline A 23 & 94 & 156 & 19 & 5 & & 0.3431 & 0.5693 & 0.0693 & 0.0182 \\
\hline A 24 & 67 & 183 & 17 & 7 & & 0.2445 & 0.6679 & 0.0620 & 0.0255 \\
\hline A 25 & 37 & 85 & 141 & 11 & & 0.1350 & 0.3102 & 0.5146 & 0.0401 \\
\hline A 31 & 31 & 123 & 104 & 16 & & 0.1131 & 0.4489 & 0.3796 & 0.0584 \\
\hline A 32 & 89 & 161 & 21 & 3 & \multirow{4}{*}{ R3 } & 0.3248 & 0.5876 & 0.0766 & 0.0109 \\
\hline A33 & 55 & 96 & 114 & 9 & & 0.2007 & 0.3504 & 0.4161 & 0.0328 \\
\hline A 34 & 38 & 152 & 72 & 12 & & 0.1387 & 0.5547 & 0.2628 & 0.0438 \\
\hline A41 & 42 & 155 & 64 & 13 & & 0.1533 & 0.5657 & 0.2336 & 0.0474 \\
\hline A42 & 202 & 70 & 2 & 0 & \multirow{2}{*}{ R4 } & 0.7372 & 0.2555 & 0.0073 & 0.0000 \\
\hline A43 & 45 & 155 & 62 & 12 & & 0.1642 & 0.5657 & 0.2263 & 0.0438 \\
\hline
\end{tabular}

According to the formula, the fuzzy comprehensive evaluation $\mathrm{T}_{\mathrm{Ai}}$ of the criterion layer and the fuzzy comprehensive

evaluation $\mathrm{T}_{\mathrm{A}}$ of the target layer can be obtained

Due to $0.5203>0.3375>0.1225>0.0197$, According to the principle of maximum degree of membership, the evaluation set

$$
\begin{aligned}
\mathrm{T}_{\mathrm{Ai}}= & \left(\begin{array}{cccc}
0.3212 & 0.5858 & 0.0783 & 0.0147 \\
0.4160 & 0.4926 & 0.0792 & 0.0122 \\
0.2231 & 0.5174 & 0.2278 & 0.0320 \\
0.3090 & 0.4841 & 0.1727 & 0.0343
\end{array}\right) \\
\mathrm{T}_{\mathrm{A}} & =W_{i} \cdot \mathrm{T}_{\mathrm{Ai}}=\left(\begin{array}{llll}
0.3375 & 0.5203 & 0.1225 & 0.0197
\end{array}\right)
\end{aligned}
$$

corresponding to the company's agricultural product supply chain resilience level of 0.5203 is good, which indicates that the company's supply chain has good resistance and recovery capabilities when risk disturbance occurs.

From the total weight of each indicator in the indicator layer in Figure 1, the main factors affecting the resilience of the agricultural product supply chain are the reliability of suppliers, the number of transport vehicles, and the risk control ability of employees. These three factors further improve the resilience of the agricultural product supply chain the key factor. When selecting a supplier, an enterprise can comprehensively consider its reliability from the supplier's productivity, on-time product delivery rate, product qualification rate, etc.; determine the optimal number of transport vehicles according to the overall operating conditions of the enterprise; at the same time pay attention to the development of employees Training can greatly improve the risk control capabilities of employees; in addition, companies also need to improve the number of indicators that are rated as medium or poor to comprehensively improve the flexibility of the agricultural product supply chain.

\section{Conclusion}

This paper uses the AHP-FCE method to construct an evaluation index system for the resilience of the agricultural product supply chain, and processes the factors that restrict each other and influence each other and cannot be described only in a quantitative manner; use the Delphi expert consultation method to give a comparison judgment matrix, and pass Quantitative mathematical calculations obtain the weight of each indicator. The results show that the main factors affecting the resilience of the agricultural product supply chain are the reliability of suppliers, the number of transportation vehicles and the risk control ability of employees; the introduction of fuzzy mathematics theory to establish a fuzzy relationship matrix is effective A fuzzy comprehensive evaluation of the resilience of the agricultural product supply chain is carried out, and corresponding optimization suggestions are put forward to provide a theoretical basis for improving the resilience of the agricultural product supply chain.

\section{References}

1 Pettit, T.J., Croxton, K.L. and Fiksel, J. Ensuring supply chain resilience: development and implementation of an assessment tool. [J] Journal of Business Logistics, 2013. 34(1): 46-76.

2 Soni, U., Jain, V. and Kumar, S. Measuring supply chain resilience using a deterministic modelling approach. [J] Computers \& Industrial Engineering, 2014. 74: 11-25.

3 Hassini, E., Surti, C., \& Searcy, C. A literature review and a case study of sustainable supply chains with a focus on metrics. [J] International Journal of Production Economics, 2012. 140(1): 69-82.

4 Joshua Aboah, Mark M.J. Wilson, Karl M. Rich, et al. Operationalising resilience in tropical agricultural value chains. [J] Supply Chain Management: An International Journal 2019, 24(2): 271-300.

5 Hosseini, S., D. Ivanov, and A. Dolgui. Review of quantitative methods for supply chain resilience analysis. [J] Transportation. 2019. 125: 285-307. 